\title{
Hiatal hernia
}

\author{
JAMES S. DAVIDSON
}

The Royal Infirmary, Bradford

INGUINAL hernia, so beautifully illustrated and animatedly discussed almost 200 years ago, still has a very definite recurrence rate after repair. In the repair of both inguinal and hiatal hernia room has to be left for the passage of an anatomical structure, the spermatic cord and the oesophagus respectively, through a muscular opening without subjecting that structure to compression. After repair of the hernia an opening remains which is a potential source of recurrence. In hiatal hernia the problem is more complicated in that we are dealing not only with the protrusion of an abdominal viscus but with a very delicate and only partially understood valvular mechanism at the oesophago-gastric junction with a boiling cauldron of acid on one side of it and a vulnerable mucosa on the other. Surgery is eminently successful in the relief of obstructions whether they be in bowel, in the biliary or urinary tract, or in a heart valve. It is far less successful in the reconstruction of one-way valvular mechanisms where co-ordinated muscle action plays such an important part. I make this preliminary comment in order to explain why any surgeon claiming $100 \%$ success in the treatment of hiatal hernia is a prevaricator and any physician expecting him to achieve such success is an uncritical optimist.

Having said this, I must hasten to add that, in my opinion, the results of repair of uncomplicated hiatal hernia are good. The question immediately arises as to what criteria are to be used to judge results. In the case of inguinal hernia success or failure may be decided merely by asking the patient to cough. In hiatal hernia, one has to assess results on symptoms or postoperative radiological findings or both. Herein lies a difficulty. It is well recognized that, although clinical success may be achieved in 80 $90 \%$ of patients, the radiological cure-rate is much lower. Pearson \& Gray (1967) reported $83 \%$ clinical successes as against radiological success in only $40 \%$. Raphael et al. (1965) said that 'although most reports indicate that the majority of patients experience symptomatic improvement after operation, rates of recurrence based on roentgenographic evidence vary from
1 to $48 \%$ '. Although this discrepancy between symptomatic and radiological results is disconcerting, I think that, for practical purposes, it is the patient's assessment of the operation which is the important thing. In the case of an internal hernia, her heart does not grieve for what her eye does not see.

The results of hiatal hernia repair depend to a large extent on case selection. Rex et al. (1961) claimed that in a 10-year study of medically treated patients, $82 \%$ with minimal symptoms improved on medical therapy alone. The very best surgical results are to be obtained by operating on patients who do not really require surgery. From what I have read in the literature, there is a tendency to describe the results of surgery in hiatal hernia while failing to detail the types of hernia operated on or to mention, almost as an aside, that complicated cases associated with stricture have been excluded from the series. I think that the results of surgery must be subdivided into those obtained in uncomplicated hernias and those obtained in the presence of the various complications of hiatal hernia, especially peptic stricture, which adversely affect the rate of cure.

The incidence of hiatal hernia in the general population is unknown. All reported series have undergone some selection. I have chosen a highly selected group of patients, namely those I have operated on in the last 3 years. In spite of the difficulties inherent in attempting to draw conclusions from such a series, I have felt that you would prefer opinions based on actual case records rather than vague impressions as seen through a surgeon's rose-coloured spectacles.

The series consists of 117 patients. Of these, eighty-one may be classified as uncomplicated hernias. By this I mean reducible hernias either sliding or para-oesophageal, the sliding hernias being usually associated with oesophagitis but without evidence of stricture at oesophagoscopy. Thirty-six patients are classified as complicated.

\section{Complications}

No less than twenty-nine had peptic stricture of the oesophagus. Two presented as strangulated 
hernia, two were associated with an oesophageal web, two with a penetrating ulcer at the level of the hiatus, and in one there was an achalasia of the cardia in addition to the hiatal hernia.

About peptic stricture I shall say more later but at this stage I would like to say a word about some of the other complications.

(1) Of the strangulated hernias, one, a man of 58, came to hospital late and died of pre-renal uraemia. One patient who presented with pain and vomiting was thought from the radiological appearances to have a strangulated hiatal hernia. There was indeed a hernia but the symptoms and $\mathrm{X}$-ray appearances were produced by pyloric stenosis and eventration of the left dome of the diaphragm.

(2) The association of upper oesophageal web with hiatal hernia was described by Smiley, McDowell \& Costello (1963). Such webs are easy to miss radiologically and, without oesophagoscopy, the dysphagia may be attributed to the hernia. In one patient there were two oesophageal webs, an upper and a lower. The upper one was split through the oesophagoscope and the lower one was excised at thoracotomy. Incidentally, lower webs are easy to miss even at oesophagoscopy. They are extremely mobile and move down in front of the oesophagoscope. At thoracotomy, they may be difficult or impossible to feel. On one occasion, when operating on a lower web and being unable to feel it, $I$ had the anaesthetist pass a large stomach tube. The tube appeared to pass well down into the stomach and, at first, I thought the diagnosis was wrong. On closer inspection, it was found that the tube had pushed the web down ahead of it. I have seen exactly the same thing in a more recent case. I have never seen this happen with a peptic stricture so that I tend to support the view that, though such webs are usually associated with a hiatal hernia, they are not peptic strictures. The web is readily removed by submucosal dissection.

(3) In the patient with a hernia and achalasia of the cardia, all the symptoms are relieved by Heller's operation and repair of the hernia (Fig. 1).

The figure of thirty-six complicated hernias does not include those associated with duodenal ulcer, disease of the biliary tract, or diverticulitis regarding which my figures are imprecise. However, I hope to have shown how frequently complications exist which must inevitably affect the results of surgery.

\section{Indications for surgery}

The high incidence of complicated cases in this series may be a reflection of a certain restraint which has been exercised in the selection ofo patients for surgery and this brings me to the question of case selection.

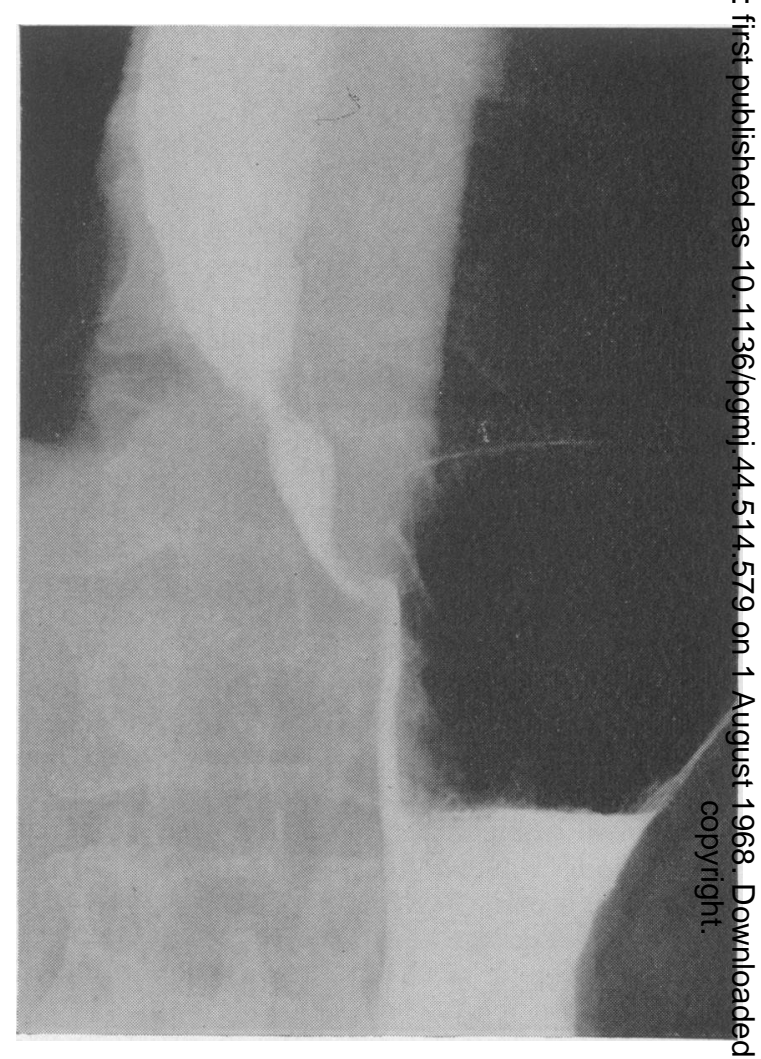

FIG. 1. Achalasia of the cardia associated with hiatal hernia.

(1) The most common indication for surgery? was the persistence of distressing heartburn and acid regurgitation in spite of medical treatment 3 . Such treatment was based largely on alkalis ando weight reduction. Getting patients in this group. to slim is difficult. One should be on the lookout for endocrine causes of obesity. I have had occasional patients with myxoedema whose heartburn was best relieved by treatment with $\rightarrow$ thyroxin. Conversely, symptoms may be initiated으․ or aggravated by weight gain under steroid ther- $\bar{N}$ apy. Regarding posture, I think it is not always realized how high above the cardia is the fluid? level in the stomach in the recumbent posture $N$ owing to the very different planes of the oesophagus and stomach (Fig. 2). Although propso ping the patient up may reduce the fluid column below the critical level of pressure necessary to ${ }^{?}$ cause reflux, the patient certainly has to be $\frac{0}{0}$ almost bolt upright before the fluid level in the $\frac{\vec{\Phi}}{\mathbb{\Phi}}$ 
stomach is at the level of the cardia. Occasionally, I think it is worth while trying to get the patient to sleep face down (Fig. 3). An offender in increasing reflux is the Goldthwaite belt for sore back. Sometimes the belt has become a habit rather than a necessity and it may be discarded with benefit.

(2) Secondly, I have taken as an indication for surgery the persistence of heartburn with, in addition, dysphagia due to acute oesophagitis

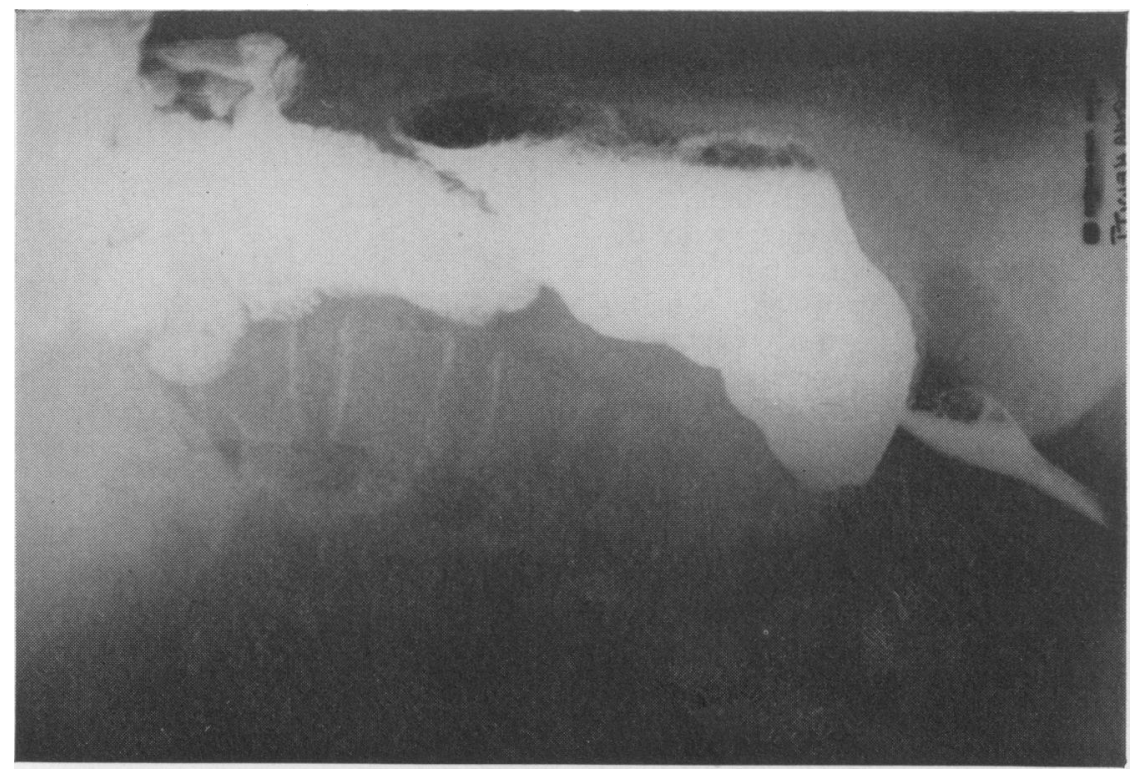

FIG. 2. Lateral X-ray with the patient lying on her back showing the height of the barium column in the stomach above the level of the cardia.

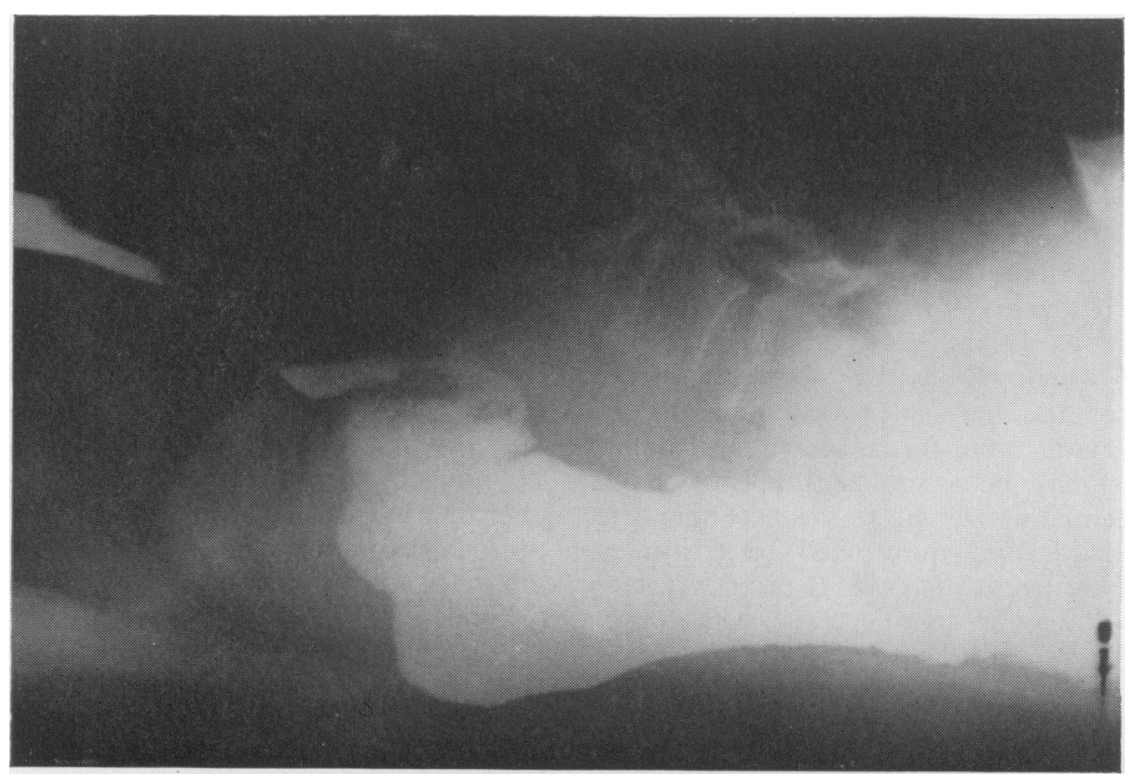

FIG. 3. Lateral X-ray with the patient lying face down showing the oesophagus lying above the barium in the stomach. 
as seen at oesophagoscopy but without stricture formation. There were twelve such patients. Rightly or wrongly, it was felt that operation in such patients might prevent progressive changes in the deeper layers of the oesophageal wall leading to peptic stricture.

This raises the problem of when repair of a hernia should be advised as a prophylactic measure against peptic stricture. In practice, the patient with a peptic stricture usually presents as such. Only occasionally does one witness by serial X-rays the development of a stricture in an initially uncomplicated hernia. I think, however, that acute oesophagitis together with discomfort on swallowing is an indication for surgery and also I think that a baby with a hernia or gastro-oesophageal reflux who regurgitates blood-stained fluid should be operated on.

(3) Thirdly, strangulation is an obvious indication for surgery. A history of episodes of pain often relieved by vomiting in the presence of a large hernia is, I think, an indication to operate in order to avert strangulation. The most dangerous type of hernia in this respect is the one which contains about half the stomach and in which a persistent fluid level is seen on the straight $X$ ray of chest, especially the lateral view. Paradoxically, when all the stomach is in the chest, the risk of strangulation, though present, seems to be less, presumably due to the fact that the portions of bowel entering and leaving the hiatus are less bulky.

(4) Another lesion demanding surgery is the peptic ulcer which goes on penetrating remorselessly and may involve the pericardium or the aorta. Such ulcers may be at or near the squamocolumnar mucosal junction, on the lesser curvature at the level of the hiatus, in the herniated portion of stomach or in a gastric-lined oesophagus. In this series there were two patients with an ulcer at hiatal level (Fig. 4). These ulcers often bleed. They are of interest as I believe that the only treatment they require is repair of the hernia which moves the ulcer away from the margins of the hiatus (Davidson, 1958).

(5) A fifth indication for surgery is bleeding. Haematemesis may be a sufficient indication on its own. Anaemia presumed to be secondary to a hernia may respond quite well to treatment with iron but recurrent anaemia is often a factor influencing the decision to operate.

(6) Lastly, in this series, peptic stricture of the oesophagus was a frequent indication for surgery.

To recapitulate, the indications for surgery were failure of medical treatment, acute oesophagitis and dysphagia without actual stric- ture formation, strangulation or a history of episodes of pain with a large hernia, penetrat- $\frac{3}{8}$ ing ulcer, haematemesis and sometimes recurrent $\stackrel{\triangleright}{\unrhd}$

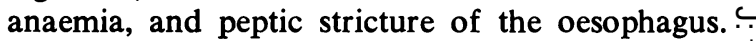

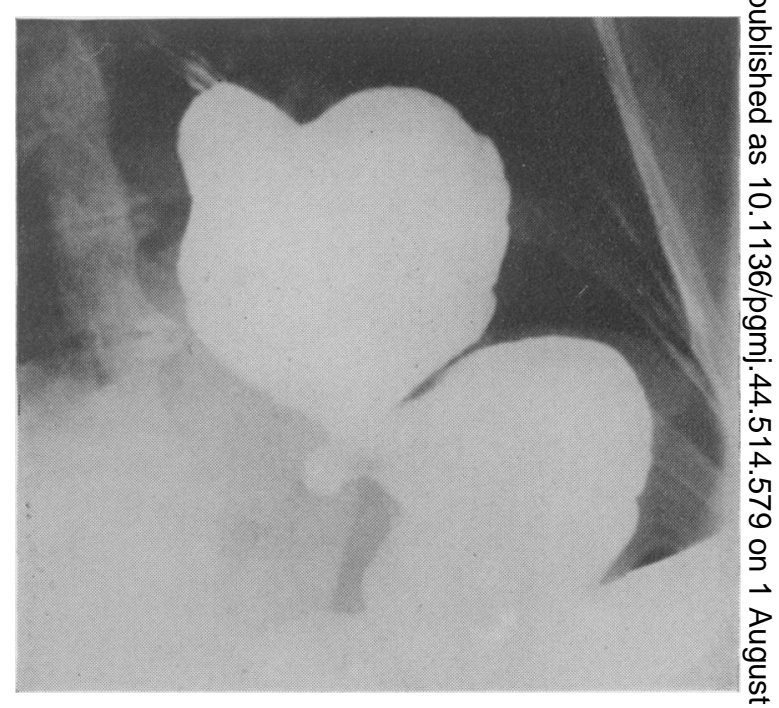

Fig. 4. A gastric ulcer at the level of the hiatus.

\section{Peptic stricture}

This is a large problem. As I have said, there were twenty-nine strictures in this series. This figure does not include all the peptic strictures I have seen in the past 3 years as, because of my approach to the management of this lesion, a number did not come to surgery. I am afraid that $I$ am in a somewhat bemused state concerning the correct treatment of this lesion. Perhaps I may illustrate my difficulties by describing some types of stricture dealt with. Peptic strictures seem to me to fall into three groups from the clinical point of view.

(1) Firstly, there is the stricture which, at oesophagoscopy looks inflamed and oedematous and feels relatively soft and mobile. This is the type of stricture which, theoretically, should respond well to control of reflux by repair of the hernia. In practice, the behaviour of such strictures is somewhat unpredictable. In this series, there are instances of at least three types of response.

(a) Patients in whom successful repair of the hernia was achieved with disappearance of the stricture.

(b) Patients in whom the hernia recurred soon after operation but in whom the dysphagia was nevertheless relieved (Fig. 5). 


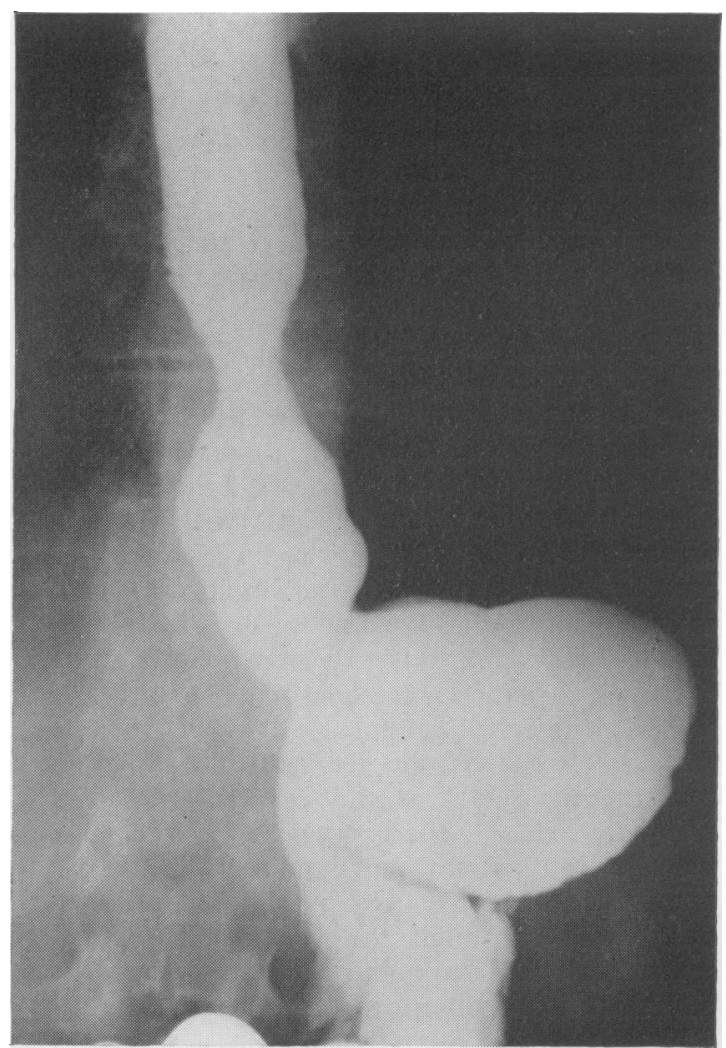

Fig. 5. Barium swallow showing recurrence of hernia in a patient whose dysphagia was nevertheless relieved.

(c) Cases where neither successful repair of the hernia nor relief of the dysphagia was achieved (Fig. 6).

(2) A second type is the high stricture with a segment of columnar-lined oesophagus above the hernia. There were at least seven such patients. They again respond in different ways to hernial repair. Some were cured. Others were converted from a long inflamed stricture to smooth, rather web-like strictures which, in contra-distinction to their pre-operative state, were much more amenable to dilatation (Fig. 7). Some remained as tight as ever.

I am obviously unable to distinguish between strictures which are going to respond to repair of the hernia and those which are not.

(3) Then there is a group of very hard strictures which neither respond well to dilatation nor disappear after attempted repair of the hernia. Three such strictures in this series developed after an operation for duodenal ulcer. This type of stricture is, I think, a very definite entity. It is possibly related to the stricture which may follow soon after delivery especially in hyperemesis gravidarum as described by Abbey Smith \& Nelson (1965). Dysphagia usually comes on about 3 weeks after an operation, often an operation for duodenal ulcer but also after a variety of other procedures. A hiatal hernia has not necessarily been present before the operation. My own view is that this stricture is due to an acute acid burn of the oesophagus during recumbency under anaesthesia. Just as it is recommended that alkali be given by mouth before anaesthesia for labour in order to prevent Mendelson's syndrome, so, I think, we may come round to the policy of giving alkali immediately before operation on patients known to have a hiatal hernia or who have a peptic ulcer or hyperchlorhydria. The following case record is typical of this type of stricture:

Perforation of a chronic duodenal ulcer occurred in a man of 32 . The perforation was closed. Three weeks later he developed dysphagia which became severe and led to emaciation and dehydration. The barium swallow showed the tapering column of barium characteristic of this

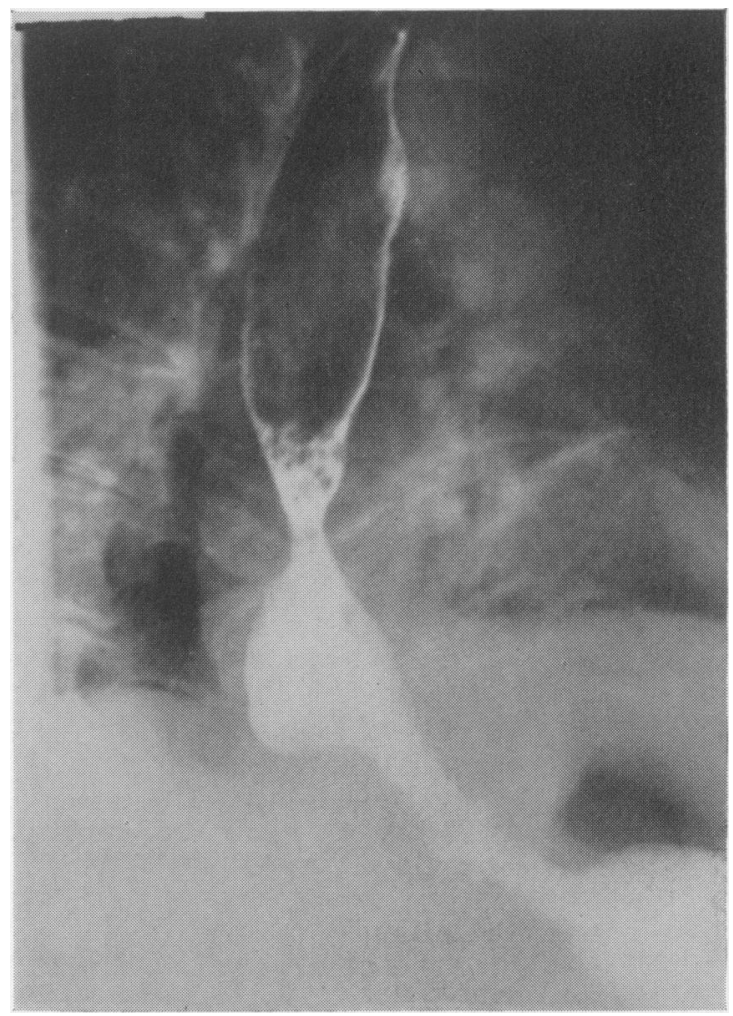

Fig. 6. Barium swallow showing recurrence of hernia where dysphagia persisted. 
stricture. The patient has done well for 2 years on self-bouginage. It is of interest-that, although no hiatal hernia was seen at several previous $\mathrm{X}$-rays over a period of some years, there is now a hernia. It is debatable whether a hernia played a part in the occurrence of stricture or whether it was, in fact, secondary to severe changes in the oesophageal wall produced by ulceration and oesophagitis.

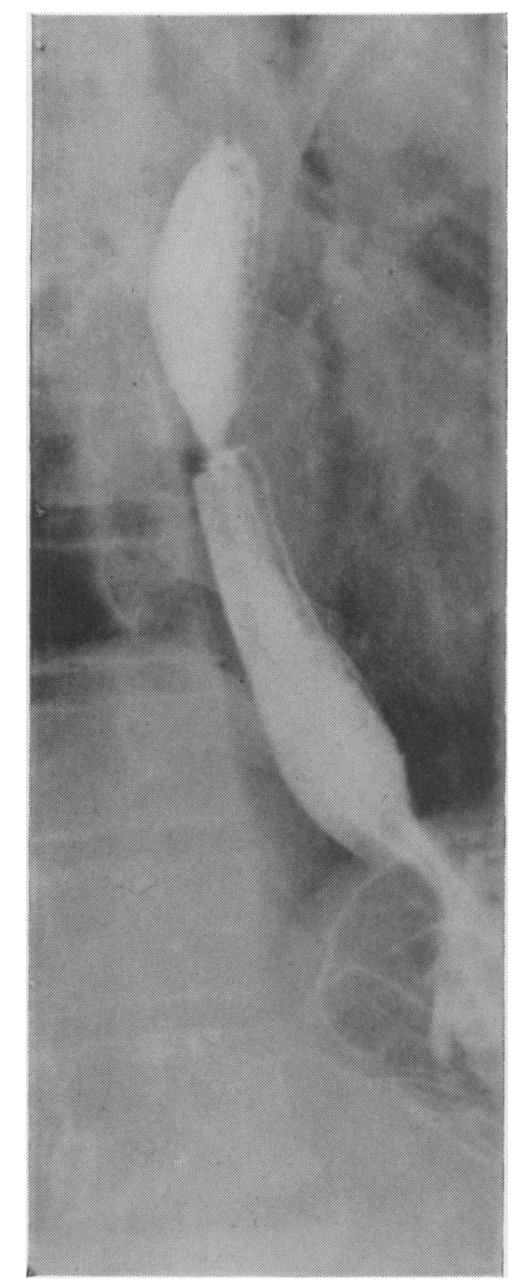

Fig. 7. Barium swallow showing a web-like stricturepersisting after repair of a hiatal hernia associated with a gastric-lined oesophagus. The stricture had previously been long and inflamed.

Of the twenty-nine strictures, relief of dysphagia by reduction of the hernia was achieved in eighteen, improvement in five, and in six the stricture remained as tight as before. In view of these figures, I think that what has been called a 'hopeful' repair of the hiatal hernia associated with stricture is often justified especially as there is a great difference in the severity of the operation between simple reduction of the hernia and excision of the stricture with colon or jejunal replacement.

My own attitude towards peptic stricture is as follows but this is a strictly personal view. I try to reduce the hernia if at all possible. There is no doubt that in a number of patients where the $\mathrm{X}$-ray appearances and the findings at oesophagoscopy seen ominous, the hernia may be easier to reduce than one might expect. This is particularly so when there is a high stricture with a gastric-lined oesophagus when the actual stricture may be easy to reduce. I have not practised Collis' operation to any extent. If the hernia seems quite irreducible or the patient is unfit or very old, I try to manage the case by dilatation before considering excision and replacement with colon or jejunum. Dilatation via the oesophagoscope gets rather a bad press. I would emphasize that, if repeated dilatation is going to succeed, there must be a close liaison between the patient and the surgeon. It is no use dilating the stricture and sending the patient away to go through the laborious business of seeking a further appointment through the usual channels. The patient must be able to ring upo and arrange for dilatation within a day or two of recurrence of dysphagia. Also, with a co-operative patient, I am sure that self-bouginage has a very definite place.

Sometimes there is no alternative to excision of the stricture but, in practice, I do few such operations for this lesion. It is indicated, I think, for a very hard stricture not responding to dilatation or where there is a penetrating ulcer which is progressive. A peptic stricture in childhood which does not respond to repair of the hernia and where the child fails to thrive and remains seriously underweight is, I think, an indication for excision of the stricture and colon replacement.

Peptic stricture of the oesophagus may undoubtedly undergo malignant change. One patient of mine had had a peptic stricture since childhood and developed a carcinoma at the site of stenosis at the age of 42 . How much one should be influenced in treatment by the possibility of malignant change I do not know.

\section{Anaemia}

In this series there were sixteen patients with significant anaemia. Six of these had haematemesis. In one patient, although there was a hernia, the anaemia was due to bleeding from a 
leiomyoma of the stomach. In two patients bleeding occurred from an ulcer at the level of the hiatus. In nine patients in whom the anaemia was presumed to be related to the hernia, the hernia was invariably a fairly large one and its repair resulted in cure of the anaemia in seven.

\section{Results}

In the hernias I have classified as uncomplicated $\mathbf{7 7 . 5} \%$ were asymptomatic after hernial repair, $15 \%$ were improved, and $7.5 \%$ were no better. When there were complications, especially stricture, only $61 \%$ were asymptomatic, $25 \%$ were improved, and as many as $14 \%$ were no better.

The main reasons for poor results were persistence of reflux or recurrence of the hernia, and persistence of dysphagia. Results were poor in a group not always mentioned but which I would describe as instances of wrong diagnosis or ill-advised surgery. It is only too easy to blame symptoms on a blameless hernia. When, after operation, some patients, as in this series, express dissatisfaction because of buzzing in the ears, nightmares, numbness round the ankles and black dust in the water, one realizes that one should never have operated on them at all.

\section{Discussion}

I have purposely avoided discussion of surgical technique. My own preference is for the transthoracic or thoraco-abdominal approach as I find it difficult to know how otherwise one can cope with the considerable number of patients with stricture and peri-oesophagitis. However, many believe otherwise and discussion of this matter is singularly unrewarding. I must admit that postoperative wound pain, which may on occasion, be severe and persistent, is a disadvantage of the thoracic approach. There is no time to describe my own not altogether successful efforts to solve this particular problem.

I cannot understand those who would undertake the surgical treatment of hiatal hernia without routine oesophagoscopy. Even the best radiologist cannot reveal all. At oesophagoscopy, one can see the site of the oesophago-gastric junction, get an idea of the function of the cardia and certainly see the moderate and severe degrees of oesophagitis with superficial erosions of the squamous mucosa. One can find a gastric- lined oesophagus not apparent on X-ray. One can determine the level of the stricture and if it is hard or soft, fixed or mobile. One can take a biopsy to exclude neoplasm. Inevitably one will discover from time to time associated lesions such as webs which have been missed on X-ray.

In spite of all $I$ have said concerning case selection, I am sure that in many instances we know not what we do. The oesophagus is a very emotional organ. In no surgery that I practise do psychological factors play such a big part. The fat woman of 55 who has, as they say, "let herself go', who no longer feels needed by her grown-up family and her disinterested husband, may seek comfort from the priest, the psychiatrist, the beautician, or the surgeon. Equally baffling is the woman with the doting husband who does all the chores. We claim cure of heartburn by repair of a hernia when all we may have done is to provide circumstances in which a woman who feels neglected may again become an object of sympathy. We accept blame for failures when the symptoms are in fact related to business worries or domestic disharmony. The dermatologist must be a psychologist and so must the oesophageal surgeon. It is very difficult to know when an oesophagus is being digested by acid and when it is blushing for shame, pining for love, quivering with anxiety, desolate with grief, or merely reflecting downward trends on the stockmarket.

I hope that I have succeeded in emphasizing the complexity of the problem, at least as far as I am concerned, while at the same time showing that the results of surgery in properly selected patients are good.

\section{References}

Abbey Smith, R. \& Nelson, C.S. (1965) Oesophageal obstruction following hyperemesis gravidarum. Thorax, 20, 528.

DAvidson, J.S. (1958) Gastric ulcer in association with hiatal hernia. Lancet, ii, 729.

Pearson, J.B. \& GraY, J.G. (1967) Oesophageal hiatus hernia: long term results of the conventional thoracic operation. Brit. J. Surg. 54, 530.

Raphael, H.A., Ellis, F.H., Carlson, H.C. \& Anderson, H.A. (1965) Surgical repair of sliding oesophageal hiatal hernia. Arch. Surg. (Chic.), 91, 228.

ReX, J.C., Anderson, H.A., Bartholomew, L.G. \& CaIn, J.C. (1961) Oesophageal hiatal hernia-a 10 year study of medically treated cases. J. Amer. med. Ass. 178, 271.

Smley, T.B., McDowell, R.F.C. \& Costello, W.T. (1963) Sideropenic dysphagia and hiatus hernia. Lancet, ii, 7. 